\title{
CATASTROPHIC ANTIPHOSPHOLIPID SYNDROME: CASE REPORT
}

\author{
Tâmara Santos Melo르. Robson Antônio Gonçalves ${ }^{1}$, Valéria Bezerra da Silva ${ }^{1}$, Gabriela Almeida Barbosa ${ }^{1}$, Danielle Christinne \\ Soares Egypto ${ }^{1}$, Maria Roberta Melo Pereira Soares ${ }^{1, \star}$, Ana Karla Guedes de Melo ${ }^{1}$, Sandra Rejane Cabral Batista ${ }^{1}$, Alessandra \\ de Sousa Braz ${ }^{1}$, Eutilia Andrade Medeiros Freire ${ }^{1}$ \\ 1.Universidade Federal da Paraíba, João Pessoa (PB), Brazil. \\ *Corresponding author: mariarobertam@hotmail.com
}

\section{BACKGROUND}

Catastrophic antiphospholipid syndrome (APS) is a subset of APS that affects approximately $0.8 \%$ of patients with primary APS. It is diagnosed by the occurrence of three or more thrombosis of new organs in one week, biopsy of small vessels with micro thrombus and previous APS and/or presence of antiphospholipid antibodies (ApL). The presence of a systemic inflammatory response with excessive release of pro-inflammatory cytokines suggests a potential thrombus-former in APS. The treatment of catastrophic APS consists in therapeutic anticoagulation, and additional therapies, such as high-dose corticosteroid therapy, plasmapheresis and intravenous immunoglobulin (IGGEV) to suppress the cytokine cascade.

\section{CASE REPORT}

Female, 25 years old, with a previous history of ischemic stroke not investigated at 19 years old. Admitted in May 2021 with leftsided hemiparesis, aphasia, fever of undetermined origin and kidney injury with oliguria, creatinine 11.71 and protein/creatinine ratio of 12.6. Started renal replacement therapy with dialysis. Diagnosed with ischemic stroke by brain angioresonance: acute encephalic ischemia in the supratentorial brain parenchyma and in the irrigation territories of the middle cerebral arteries with more extensive findings on the right, multiple areas of intracranial arterial vascular narrowing in all extension of the anterior cerebral arteries and middle cerebral arteries. Given the suspicion of catastrophic APS, abdominal computed tomography angiography (CTA) showed mural thrombus in the distal abdominal aorta, thrombotic occlusion of the left common iliac artery and areas of acute microhemorrhage in the left renal parenchyma. Chest CTA showed ground-glass alveolar opacities, affecting approximately $50 \%$ of the parenchyma, bilateral, peripheral and peribronchovascular, predominantly in the lower lobes, suggestive of alveolar hemorrhage. Laboratory tests: quasi-homogeneous nuclear ANA 1:320, anticardiolipin IgM: 21.5 MLP and lgG: 84.9 GLP, complement consumption (C3: 88, C4: 7.9). The RT-PCR for COVID-19: negative. Treated with pulse therapy with methylprednisolone $1 \mathrm{~g} /$ day for 5 days, IGGEV $400 \mathrm{mg} / \mathrm{kg} / \mathrm{day}$ for 5 days due to fever peaks and unavailability of plasma exchange. Because of the renal condition, a dose of cyclophosphamide $1 \mathrm{mg} / \mathrm{m}^{2} / \mathrm{SC}$ corrected for renal function was performed. The patient underwent prophylactic enoxaparin and, 14 days after the stroke, warfarin was started. After 16 days of the ischemic event, she evolved with cerebral hemorrhagic transformation and death.

\section{CONCLUSION}

Catastrophic APS is frequently fatal, with a mortality rate close to $50 \%$ in diagnosed patients, despite anticoagulant and immunosuppressive treatment. Indicating the need for early diagnosis and adequate follow-up of the APS.

\section{KEYWORDS}

Catastrophic antiphospholipid syndrome, Antiphospholipid antibodies, Catastrophic APS, Microthrombus. 